Electronic version of an article published as [Lecture Notes in Computer Science, 2007, No. 4705, p. 791-803] [DOI: http://dx.doi.org/10.1007/978-3-540-74472-6_65]

(C) [copyright Springer Verlag] 


\title{
Evaluating optimization models to solve SALBP*
}

\author{
Rafael Pastor, Laia Ferrer, Alberto García \\ Technical University of Catalonia, IOC Research Institute, Av. Diagonal 647, Edif. \\ ETSEIB, p.11, 08028 Barcelona, Spain \\ \{rafael.pastor, laia.ferrer, alberto.garcia\}@upc.edu
}

\begin{abstract}
This work evaluates the performance of constraint programming (CP) and integer programming (IP) formulations to solve the Simple Assembly Line Balancing Problem (SALBP) exactly. Traditionally, its exact solution by CP or IP and standard software has been considered to be inefficient to real-world instances. However, nowadays this is becoming more realistic thanks to recent improvements both in hardware and software power. In this context, analyzing the best way to model and to solve SALBP is acquiring relevance. The aim of this paper is to identify the best way to model SALBP-1 (minimizing the number of stations, for a given cycle time) and SALBP-2 (minimizing the cycle time, for a given number of stations). In order to do so, a wide computational experiment is carried out to analyze the performance of one CP and three IP formulations to solve each problem. The results reveal which of the alternative models and solution techniques is the most efficient to solve SALBP-1 and SALBP-2, respectively.
\end{abstract}

Keywords: assembly line balancing

\section{Introduction}

An assembly line consists in set of workstations, through which the product to be processed flows. In each workstation, a number of tasks are done, which are characterized by their processing times and by a set of technological precedence relations between them. The Simple Assembly Line Balancing Problem (SALBP) consists of assigning a set of tasks to workstations in such a way that precedence constraints are fulfilled, the total processing time assigned to a station do not exceed a cycle time tc and a given efficiency measure is optimized. When the objective is to minimize the number of workstations $m$ for a given cycle time $t c$, the problem is usually referred to as SALBP-1; if the objective is to minimize tc given $m$, the problem is called SALBP-2; and SALBP-F consists of finding a feasible solution, given $t c$ and $m$ (see e.g. [1]).

The design of assembly lines has been extensively examined in the literature, especially the SALB Problem. Several reviews have been published -the last is [2]-, and a huge amount of specific research exists, both for heuristic and exact procedures.

* This work is supported by the Spanish MCyT project DPI2004-03472, co-financed by FEDER. 
Some of the exact procedures are based on mathematical programming and different integer linear programming and mixed-integer linear programming models (IP models) have been developed. Scholl highlights three basic formulations to solve SALBP-F [1] (finding a feasible solution, given a cycle time and a number of stations) based on different sets of assignment variables. Other exact procedures have also used constraint programming (CP) [3].

Recent improvements both in software and hardware power have reduced remarkably the computing time needed to solve combinatorial problems by constraint programming or mathematical programming. Nowadays, these techniques are gaining acceptance as a powerful computational tools [4]. In this context, analyzing the best way to model and to solve combinatorial problems is acquiring relevance. Constraint programming and mathematical programming can solve similar combinatorial problems, but their effectiveness depends on the class of problems studied [5]. A number of papers have compared the performance of CP and IP approaches for solving different problems - for example, [6].

To our knowledge, the efficiency of a CP model and the IP enhanced by Scholl [1] models has not been compared. In this work, a wide computational experiment is carried out to analyze the performance of one CP model and three IP models to solve SALBP-1 and SALBP-2. The results reveal which of the alternative models is the most efficient to solve these SALBP problems.

The remaining paper is organized as follows. In Section 2 the different formulations for SALBP-1 and SALBP-2 are presented. In Section 3 the results of the computational experiment are analyzed and the performances of the models are compared. Finally, in Section 4 the main conclusions of the study are summarized.

\section{Models for SALBP}

In this section four alternative formulations for SALBP-1 and SALBP-2 are developed.

First, we present a CP model - constraint programming model-.

Then, the three SALBP-F models presented in Scholl (1999) are adapted to SALBP-1 and SALBP-2. In sum, the main difference between these three linear models is the definition of the assignment variables used:

- impulse variables based model: binary variables $x_{i j}$ take value 1 if and only if task $i$ is assigned to workstation $j$ (see also [7] and [8])

- step variables based model: binary variables $x_{i j}$ take value 1 if and only if task $i$ is assigned to workstation $j$ or earlier (see also [7] and [8]).

- mixed-integer variables model: integer variables $z_{i}$ denotes the number of the station to which task $i$ is assigned.

In the following sections the formulations for the four models are detailed. In each section, first the model for SALBP-1 is presented. Then the model for SALBP-2 is explained highlighting the new data, the new variables and the changes to be done in the formulation with respect to the model for SALBP-1. 


\subsection{The constraint programming models}

Next, the constraint programming model for SALBP-1 (SALBP-1-C) is presented and the changes for SALBP-2 (SALBP-2-c) are explained.

\section{SALBP-1-C}

Data:

Note subindexes $i$ and $k$ are related with tasks and subindex $j$ with workstations.

$n \quad$ Number of tasks $(i=1, \ldots, n)$.

$m_{\max } \quad$ Upper bound on the number of workstations $\left(j=1, \ldots, m_{\max }\right)$.

$m_{\min } \quad$ Lower bound on the number of workstations.

$t_{i} \quad$ Processing time of task $i$.

TC Cycle time.

$P \quad$ Set of pairs of tasks $(i, k)$ such that there is immediate precedence between them.

$S \quad$ Set of tasks without any successive task.

$E_{i} \quad$ Earliest possible workstation for task $i$.

$L_{i} \quad$ Latest possible workstation for task $i$, given a value of $m_{\max }$.

Before a task is assigned the total processing time of the tasks that precede it must be assigned, and afterwards the total time of the tasks that follow it; as a result, the range of workstations $\left[E_{i}, L_{i}\right]$ to which each task can be assigned is obtained and the number of binary variables is reduced (see, for example, [1]).

Variables:

ws Number of workstations used.

$z_{i} \quad$ Number of the workstation to which task $i$ is assigned $\left(\forall i ; z_{i} \in\left[E_{i}, L_{i}\right]\right)$

Model SALBP-1-C:

$$
\begin{gathered}
{[M I N] Z=w S} \\
w S=\max \left(Z_{i} \mid i \in S\right) \\
\sum_{\forall i \mid j \in\left[E_{i}, L_{i}\right] \wedge\left(z_{i}=j\right)} t_{i} \leq T C \quad \forall j \\
Z_{i} \leq Z_{k} \quad \forall(i, k) \in P
\end{gathered}
$$

The objective function (1) consists in minimizing the number of workstations, which is calculated in (2); constraints (3) ensures that the total task processing time 
assigned to workstation $j$ does not exceed the cycle time; constraint set (4) imposes the technological precedence conditions.

\section{$S A L B P-2-c$}

Data:

The model uses the same data of $S A L B P-1-c$; furthermore we redefine:

$m \quad$ Number of workstations $(j=1, \ldots, m)$.

$C \quad$ Upper bound on the cycle time.

$E_{i} \quad$ Earliest possible workstation for task $i$, given a value of $C$.

$L_{i} \quad$ Latest possible workstation for task $i$, given a value of $C$.

Variables:

tc $\quad$ Cycle time.

Model SALBP-2-C:

$$
\begin{aligned}
{[M I N] Z } & =t c \\
\sum_{\forall i \mid j \in\left[E_{i}, L_{i}\right] \wedge\left(z_{i}=j\right)} t_{i} & \leq t c \quad \forall j
\end{aligned}
$$

Constraint (4) has to be added.

The objective function (5) minimizes the cycle time and constraint set (6) ensures that the total task processing time assigned to workstation $j$ does not exceed the cycle time.

\subsection{The impulse variables based models}

Next, the impulse variables based model for SALBP-1 (SALBP-1-i) is presented and the changes for SALBP-2 (SALBP-2-i) are explained.

\section{SALBP-1-i}

Data:

The data used in this model is the same as the previous one.

\section{Variables:}

$x_{i j} \in\{0,1\} \quad 1$, if and only if task $i$ is assigned to workstation $j$, value 0 otherwise $\left(\forall i ; j=E_{i}, \ldots, L_{i}\right)$. 
$y_{j} \in\{0,1\} \quad 1$, if and only if any task is assigned to workstation $j$ $\left(j=\mathrm{m}_{\min }+1, \ldots, m_{\max }\right)$.

Model SALBP-1-i:

$$
\begin{gathered}
{[M I N] Z=\sum_{j=m_{\min }+1}^{m_{\max }} j \cdot y_{j}} \\
\sum_{j=E_{i}}^{L_{i}} x_{i j}=1 \quad \forall i \\
\sum_{\forall i \mid j \in\left[E_{i}, L_{i}\right]} t_{i} \cdot x_{i j} \leq T C \quad j=1, \ldots, m_{\min } \\
\sum_{\forall i \mid j \in\left[E_{i}, L_{i}\right]} t_{i} \cdot x_{i j} \leq T C \cdot y_{j} \quad j=m_{\min }+1, \ldots, m_{\max } \\
\sum_{j=E_{i}}^{L_{i}} j \cdot x_{i j} \leq \sum_{j=E_{k}}^{L_{k}} j \cdot x_{k j} \quad \forall(i, k) \in P
\end{gathered}
$$

The objective function (7) consists in minimizing the number of workstations; constraint set (8) implies that each task $i$ is assigned to one and only one workstation; constraints (9) and (10) are equivalent to (3) and they ensure the cycle time is not exceeded; constraint set (11) replaces (4) and imposes the precedence conditions.

\section{SALBP-2-i}

Data:

The data used in this model is the same as the previous ones.

Variables:

The variables have been defined in the previous models.

Model SALBP-2-i:

$$
\begin{gathered}
{[M I N] Z=t c} \\
\sum_{\forall i \mid j \in\left[E_{i}, L_{i}\right]} t_{i} \cdot x_{i j} \leq t c \quad \forall j
\end{gathered}
$$

Constraints (8) and (11) have to be added.

The objective function (5) minimizes the cycle time; constraint set (12) is equivalent to (6) and ensures that the total task processing time assigned to workstation $j$ does not exceed the cycle time. 


\subsection{The step variables based models}

Next, the step variables based model for SALBP-1 (SALBP-1-s) is presented and the changes for SALBP-2 (SALBP-2-s) are explained.

\section{SALBP-1-s}

Data:

The data used in this model is the same as the previous ones.

Variables:

The variables used in the step variables based models are the same of the impulse variables based models but $X_{i j}$ are redefined:

$x_{i j} \in\{0,1\} \quad 1$, if and only if task $i$ is assigned to workstation $j$ or earlier, 0 otherwise $\left(\forall i ; j=E_{i}, \ldots, L_{i}-1\right)$. Note that $x_{i, L_{i}}=1$ and it is not defined.

$y_{j} \in\{0,1\} \quad 1$, if and only if any task is assigned to workstation $j, 0$ otherwise $\left(j=\mathrm{m}_{\min }+1, \ldots, m_{\max }\right)$.

Model SALBP-1-s:

$$
\begin{gathered}
{[M I N] Z=\sum_{j=m_{\min }+1}^{m_{\max }} j \cdot y_{j}} \\
x_{i j} \leq x_{i, j+1} \quad \forall i ; j=E_{i}, \ldots, L_{i}-2 \\
\sum_{\forall i \mid j=E_{i}} t_{i} \cdot x_{i j}+\sum_{\forall i \mid j \in\left[E_{i}+1, L_{i}-1\right]} t_{i} \cdot\left(x_{i j}-x_{i, j-1}\right)+\sum_{\forall i \mid j=L_{i}} t_{i} \cdot\left(1-x_{i, j-1}\right) \leq T C ; \\
\sum_{\forall i \mid j=E_{i}} t_{i} \cdot x_{i j}+\sum_{\forall i \mid j \in\left[E_{i}+1, L_{i}-1\right]} t_{i} \cdot\left(x_{i j}-x_{i, j-1}\right)+\sum_{\forall i \mid j=L_{i}} t_{i} \cdot\left(1-x_{i, j-1}\right) \leq T C \cdot m_{\min } \\
\quad j=m_{\min }+1, \ldots, m_{\max } \\
x_{k j} \leq x_{i j} \quad \forall(i, k) \in P ; \forall j \in\left[E_{i}, L_{i}-1\right] \cap\left[E_{k}, L_{k}-1\right]
\end{gathered}
$$

Constraint sets (13), (14) and (15) are equivalent to constraint sets (8), (9) and (10), respectively. Now, the technological precedence conditions -constraint set (4) or (11)- is modeled by (16). 


\section{SALBP-2-S}

Data:

The data used in this model is the same as the previous ones.

Variables:

The variables have been defined in the previous models.

Model SALBP-2-s:

$$
\begin{gathered}
{[M I N] Z=t c} \\
\sum_{\forall i \mid j=E_{i}} t_{i} \cdot x_{i j}+\sum_{\forall i \mid j \in\left[E_{i}+1, L_{i}-1\right]} t_{i} \cdot\left(x_{i j}-x_{i, j-1}\right)+\sum_{\forall i \mid j=L_{i}} t_{i} \cdot\left(1-x_{i, j-1}\right) \leq t c \quad \forall j
\end{gathered}
$$

Constraints (13) and (16) have to be added. Constraint set (17) is equivalent to (6) and (12).

\subsection{The mixed-integer variables based models}

Next, the mixed-integer variables based model for SALBP-1 (SALBP-1-m) is presented and the changes for SALBP-2 (SALBP-2-m) are explained.

\section{SALBP-1-m}

Data:

The model uses the same data of the previous ones; furthermore we define:

$P^{*} \quad$ Set of pairs of tasks $(i, k)$ such that there is an immediate or transitive precedence between them.

$T \quad$ Upper-bound of the total time of the workstations.

\section{Variables:}

This formulation introduces continuous non-negative variables $b_{i}$ for the clock time at which task $i$ is started and binary variables $w_{i k}$ :

$b_{i} \in\{0,1\} \quad$ Clock time at which task $i$ is started (measured in the time elapsed since entering the first workstation).

$w_{i k} \in\{0,1\} \quad 1$, if and only if task $i$ is performed before task $k$, value 0 otherwise $\left(i<k ;(i, k) \notin P^{*} ;\left[E_{i}, L_{i}\right] \cap\left[E_{k}, L_{k}\right] \neq \varnothing\right)$.

ws $\quad$ Number of workstations used.

$z_{i} \quad$ Number of the workstation to which task $i$ is assigned $\left(\forall i ; z_{i} \in\left[E_{i}, L_{i}\right]\right)$. 
Model SALBP-1-m:

$$
\begin{gathered}
{[M I N] Z=w s} \\
w s \geq z_{i} \quad \forall i \\
b_{i} \geq T C\left(z_{i}-1\right) \quad \forall i \\
b_{i}+t_{i} \leq T C z_{i} \quad \forall i \\
\left(1-w_{i k}\right) \cdot T+b_{k} \geq b_{i}+t_{i} \quad i<k,(i, k) \notin P^{*},\left[E_{i}, L_{i}\right] \cap\left[E_{k}, L_{k}\right] \neq \varnothing \\
w_{i k} \cdot T+b_{i} \geq b_{k}+t_{k} \quad i<k,(i, k) \notin P^{*},\left[E_{i}, L_{i}\right] \cap\left[E_{k}, L_{k}\right] \neq \varnothing \\
b_{i}+t_{i} \leq b_{k} \quad(i, k) \in P, L_{i} \geq E_{k} \\
E_{i} \leq z_{i} \quad \forall i \\
z_{i} \leq L_{i} \quad \forall i
\end{gathered}
$$

The objective function (1) consists in minimizing the number of workstations calculated by constraint set (18); constraint sets (19) and (20) ensure that each task $i$ is fully performed within one workstation; the disjuntive constraints (21) and (22) guarantee that for each pair of tasks, which are not related by precedence and may interfere which each other, either task $i$ is completely processed before task $k$, or vice versa; constraint set (23) ensure the fullfilment of the precedence constraints; the assignment task is restricted to the possible workstation interval by (24) and (25).

\section{SALBP-2-m}

The adaptation of mixed-integer variables based model for SALBP-F to SALBP-2 produces a non-linear model since the variable cycle time tc replaces data $T C$ in constraints (19) and (20). This non-linear formulation of SALBP-2-m is linearised as follows.

Variables:

$p_{i} \quad$ not negative real variable that indicates the total time of the workstations until the workstation in which task $i$ is assigned (this one also included)

$r_{i j} \in\{0,1\} 1$, if and only if task $i$ is assigned to workstation $j$, value 0 otherwise $\left(\forall i ; j=E_{i}, \ldots, L_{i}\right)$.

Model SALBP-2-m:

$$
\begin{gathered}
{[M I N] Z=t c} \\
b_{i}+t c \geq p_{i} \quad \forall i
\end{gathered}
$$




$$
\begin{aligned}
b_{i}+t_{i} \leq p_{i} & \forall i \\
z_{i}=\sum_{j=E_{i}}^{L_{i}} j \cdot r_{i j} & \forall i \\
\sum_{j=E_{i}}^{L_{i}} r_{i j}=1 & \forall i \\
p_{i}-j \cdot t c \leq\left(1-r_{i j}\right) \cdot T & \forall i, j=E_{i}, \ldots, L_{i} \\
j \cdot t c-p_{i} \leq\left(1-r_{i j}\right) \cdot T & \forall i, j=E_{i}, \ldots, L_{i}
\end{aligned}
$$

The real variables $p_{i}$ replaces the product $t c \cdot z_{i}$ in (19) and (20) obtaining (26) and (27); the variables $z_{i}$ are expressed as shown in (28); constraint sets (29), (30) and (31) are added.

Constraint sets (21)-(23) need to be added too.

\section{Computational experiment}

A computational experiment is carried out to compare the efficiency of the models.

The basic data used for the experiment are all the well-known instances available in the assembly line balancing research homepage (www.assembly-linebalancing.de). A total of 269 instances for SALBP-1 and 302 for SALBP-2 were used.

The CP models were solved using ILOG Solver 6.0 and the MILP models were solved by CPLEX 9.0, with a PC Pentium IV at $3.4 \mathrm{GHz}$ and with $512 \mathrm{Mb}$ of RAM. A maximum computing time of 2,000 seconds was set.

The analysis of the results of the computational experiment starts with a initial comparison of the performance of the models in terms of the type of the solutions obtained: whether the model finds a solution or not and whether this solution is optimal or feasible. This initial analysis identifies the best models to be analyzed in detail. Next, the computing time used by these models is studied, focusing on the instances in which the optimal solution is found. Next, the solutions obtained in the instances in which the optimality is not guaranteed are presented. Finally, considering all these aspects, a detailed analysis of the performance of the different models is carried out.

\subsection{Results of the type the solutions}

Table 1 and table 2 show the results of the computational experiment for SALBP-1 and SALBP-2, respectively, focusing on the type of the solutions obtained. For each model, the following information is summarized: 
- the number of instances with a proved optimal solution (Opt-prov): an optimal solution is found and the solving software guarantees it.

- the number of instances in which an unproved optimal solution $(\mathrm{Opt}-\overline{\mathrm{prov}})$ : an optimal solution is found but the solving software does not guarantee its optimality. The optimal solution of the instances is available in the assembly line balancing research homepage.

- the number of instances with a feasible but not optimal solution $(\mathrm{Fea}-\overrightarrow{\mathrm{opt}})$.

- the number of instances in which the solving software does not find any solution $(\overline{\mathrm{Sol}})$.

Table 1. Results of the computational experiment for SALBP-1

\begin{tabular}{|c|c|c|c|c|c|c|c|c|}
\cline { 2 - 10 } \multicolumn{1}{c|}{} & \multicolumn{4}{c|}{ SALBP-1 } & \multicolumn{4}{c|}{ SALBP-2 } \\
\cline { 2 - 10 } \multicolumn{1}{c|}{} & $c$ & $i$ & $S$ & $m$ & $c$ & $i$ & $s$ & $m$ \\
\hline Opt - prov & 98 & 136 & 123 & 51 & 55 & 84 & 122 & 0 \\
\hline Opt $-\overline{\text { prov }}$ & 12 & 17 & 5 & 24 & 0 & 16 & 12 & 4 \\
\hline Fea $-\overline{\text { opt }}$ & 2 & 19 & 14 & 14 & 199 & 174 & 168 & 64 \\
\hline$\overline{\text { Sol }}$ & 157 & 97 & 127 & 180 & 48 & 28 & 0 & 234 \\
\hline
\end{tabular}

The results show that the performance of the mixed-integer based model is worse than the performance of the constraint programming model, the impulse variables and the step variables based models. For SALBP-1, SALBP-1-m obtains 51 proved optimal solutions; nearly half of the optimal solutions reached by SALBP-1-c, SALBP1-i or SALBP-1-s (98, 136 and 123, respectively). For SALBP-2, SALBP-2-m does not obtain any proved optimal solution. Moreover, the mixed-integer variables based models do not reach a feasible solution in more instances than the other models, both for SALBP-1 and for SALBP-2.

Due to clear inferiority of the mixed-integer variables based model, we focus the detailed comparison of the results only in the constraint programming, the impulse variables and the step variables based models. We analyze the percentage of proved optimal solutions depending on the the number of tasks $(N T)$ and the order strength $(O S=$ number of all precedence relations / $(N T *(N T-1)))$ of the instances. We classify: i) Low-OS $(22.49 \leq O S \leq 25.80)$, Middle-OS $(40.38 \leq O S \leq 60.0)$ and HighOS $(70.95 \leq O S \leq 83.82)$; ii) Low-NT $(7 \leq N T \leq 45)$, Middle-NT $(53 \leq N T \leq 111)$ and High-NT $(148 \leq N T \leq 297)$. Table 2 shows the percentage of proved optimal solutions obtained with the constraint programming (c), impulse variables (i) and step variables (s) based models. 
Table 2. Percentage of proved optimal solutions depending on $O S$ and NT

\begin{tabular}{|c|c|c|c|c|c|l|}
\cline { 2 - 7 } \multicolumn{1}{c|}{} & \multicolumn{3}{c|}{ SALBP-1 } & \multicolumn{3}{c|}{ SALBP-2 } \\
\cline { 2 - 7 } \multicolumn{1}{c|}{} & $c$ & $i$ & $S$ & $c$ & $i$ & $S$ \\
\hline Low-OS & 10.77 & 29.23 & 12.31 & 6.061 & 21.21 & 25.76 \\
\hline Middle-OS & 40.79 & 55.92 & 53.95 & 18.68 & 28.02 & 36.81 \\
\hline High-OS & 55.77 & 61.54 & 63.46 & 31.48 & 35.19 & 70.37 \\
\hline Low-NT & 100.00 & 98.72 & 98.72 & 77.50 & 97.50 & 100.00 \\
\hline Middle-NT & 14.62 & 43.85 & 33.85 & 10.71 & 19.90 & 36.73 \\
\hline High-NT & 1.64 & 3.28 & 3.28 & 4.55 & 9.09 & 15.15 \\
\hline
\end{tabular}

\subsection{Results of the computing time}

We compare the computing time used by the constraint programming, the impulse variables and the step variables based models when all of them obtain a proved optimal solution (95 instances in SALBP-1 and 48 instances in SALBP-2). Table 3 shows, for each model: the number of instances with the minimum calculation time (in seconds) to obtain a proved optimal solution (Best time); the total of time used by these instances (Total time); and the number of instances in which the time used by the model is less than $75 \%$ of the time used by each of the other two models (time $(a / b)<0.75)$.

Table 3. Results when the 3 models find an optimal solution.

\begin{tabular}{|c|c|c|c|c|c|c|}
\cline { 2 - 7 } \multicolumn{1}{c|}{} & \multicolumn{3}{c|}{ SALBP-1 } & \multicolumn{3}{c|}{ SALBP-2 } \\
\cline { 2 - 7 } \multicolumn{1}{c|}{} & $c$ & $i$ & $s$ & $c$ & $i$ & $s$ \\
\hline Best-time & 26 & 47 & 22 & 27 & 15 & 7 \\
\hline Total time & 2084.4 & 3302.4 & 2824.7 & 2491.3 & 6608.2 & 508.9 \\
\hline time $(a / b)<0.75$ & 9 & 1 & 2 & 8 & 0 & 5 \\
\hline
\end{tabular}

\subsection{Results of the solutions with no optimality guaranteed}

Next, we summarize the results obtained when the constraint programming, the impulse variables and the step variables based models find a feasible solution but none of them guarantees optimality. This situation occurs in 1 instance for SALBP-1, and in 119 instances for SALBP-2: in 10 of them SALBP-2-C obtains the best solution, $S A L B P-2-i$ in 9 instances and SALBP-2-S in 82. When SALBP-2-S obtains a better solution, the average solution is $95.5 \%$ and $71.1 \%$ of the average obtained by $S A L B P$ 2-i and SALBP-2-c, respectively.

\subsection{Analysis of the performance of models}

In this section, a detailed analysis of the performance of the models is carried out. First, we study the results for $S A L B P-1$ and then a similar study is presented for $S A L B P-2$. Each study starts with a brief final conclusion to facilitate the comprehension of the analysis of the results. These conclusions are justified through a 
detailed analysis that compares the type of solutions obtained, the computing time used and the results of the solutions in which their optimality is not guaranteed. Due to clear inferiority of the performance of the mixed-integer variables based model (Section 3.1), these analyses focus on the constraint programming, the impulse variables and the step variables based models.

\section{For SALBP-1:}

In sum, in terms of number of optimal and feasible solutions the results of $S A L B P-1-i$ are better than results of $S A L B P-1-c$ and $S A L B P-1-S$. However, concerning the time used, $S A L B P-1-C$ is the quickest model.

In terms of the type of solutions obtained (Table 1 ), the number of proved and unproved optimal solutions obtained by $S A L B P-1-i$ is higher than those obtained by $S A L B P-1-C$ and those obtained by SALBP-1-s (136 and 17, 98 and 12, 123 and 5, respectively). Moreover, $S A L B P-1-i$ does not obtain a feasible solution in less instances than $S A L B P-1-c$ and $S A L B P-1-s$ (97, 157 and 127, respectively) The results of $S A L B P-1-C$ are worse than the results of the other models, in particular, for instances with middle and high levels of $N T$; the performance of $S A L B P-1-i$ is especially the best for instances with low OS (Table 2).

In terms of the computing time (Table 3 ), when the three models guarantee the optimal solution, $S A L B P-1-i$ need less time in more instances than $S A L B P-1-C$ and $S A L B P-1-s$ (47, 26 and 22, respectively). Nevertheless, for solving all 95 instances the time needed by $S A L B P-1-C$ is considerably less than the time required by $S A L B P-1-i$ and by $S A L B P-1-s$ (2084.4 s, 3302.64 s and 2824.7 s, respectively). Moreover, among the 26 instances where $S A L B P-2-c$ is quicker, there the 9 instances in which the time used is less than $75 \%$ of the time needed by each of the other two models, whereas this difference only occurs in 1 instance for $S A L B P-1$-i and 2 for $S A L B P-1-C$.

\section{For SALBP-2:}

In sum, the results of $S A L B P-2-s$ are much better than the results of $S A L B P-2-C$ and $S A L B P-2-i$, in terms of optimal and feasible solutions obtained and total computing time. $S A L B P-2-C$ is only superior to $S A L B P-2-s$ in the number of instances that use the minimum time.

In terms of the type of solutions obtained (Table 1 ), the $S A L B P$-2-s model obtains more proved solutions than SALBP-2-C and SALBP-2-i: (122, 55 and 84, respectively). The total number of optimal solutions (proved and unproved) is also superior for $S A L B P-2-S$ than for $S A L B P-2-C$ and $S A L B P-2-i$ (134, 55 and 100, respectively). In addition, $S A L B P-2-s$ always obtains a feasible solution whereas $S A L B P-2-C$ does not obtain a feasible solution in 48 instances and SALBP-2-i in 28. The influence of NT is similar in the 3 models, SALBP-1-C is remarkably the best model for instances of low OS (Table 2).

In terms of the computing time (Table 3), when the three models guarantee an optimal solution, $S A L B P-2-c$ uses less time in more instances than $S A L B P-2-i$ and $S A L B P-2-s$ (27, 15 and 7, respectively). Moreover, the time used by SALBP-2-C is in 8 instances less than 75\% of the time used by the other models (SALBP-2-i does not have this difference in any instance and $S A L B P-2-S$ only in 5). However, for solving all the instances in which the three models guarantee an optimal solution, the total 
time used for $S A L B P-2-s$ is much less than the time used by SALBP-2-c and SALBP2-i (508.9 s, $2492.4 \mathrm{~s}$ and $6608.2 \mathrm{~s}$, respectively).

Finally, when none of the models guarantees the optimal solution, SALBP-2-s obtains a better solution in considerably more instances than the others.

\section{Conclusions}

The SALB Problem has been extensively examined in the literature and different and equivalent CP models and IP models have been developed in order to solve it. However, their efficiency has not been compared and the best one is not known. The best way to model and to solve the hard combinatorial problems has a high relevance. The use of constraint programming or mathematical programming techniques to solve these problems is becoming more realistic thanks to recent improvements both in software and hardware power.

This paper focus on comparing one CP formulation -constraint programming model- and three IP formulations that were highlighted by Scholl [1] -the impulse variables, the step variables and the mixed-integer variables based model-. A wide computational experiment is carried out to compare the efficiency of these models, both for SALBP-1 and SALBP-2.

The analysis of the results shows the bad performance of the mixed-integer variables models. For SALBP-1, the impulse variables based model obtains the best solutions although constraint programming model is the quickest. The step variables based model obtains the best results for SALBP-2.

\section{References}

1. Scholl, A.: Balancing and sequencing of assembly lines. Physica, Heidelberg, $2^{\text {nd }}$ edition (1999).

2. Scholl, A., Becker, C.: State-of-the-art exact and heuristic solution procedures for simple assembly line balancing. European Journal of Operational Research, Vol. 168, 666-693 (2006).

3. Bockmayr, A. Pisaruk, N.: Solving an assembly line balancing problem combining IP and CP. Proceedings of the $6^{\text {th }}$ Annual Workshop of ERCIM Working Droup on Constraints, Prague, Czech Republic (2001).

4. Atamtürk, A., Savelsbergh, M.W.P.: Integer-programming software systems. Annals of Operations Research, Vol. 140, 67-124 (2005)

5. Jain, V., Grossman, I.E.: Algorithms for hybrid MILP/CP models for a class of optimization problems. Journal on computing, Vol. 13 (4), 258-276 (2001).

6. Darby-Dowman, K., Little, J.: Properties of some combinatorial optimization problems and their effect on the performance of integer programming and constraint logic programming. Journal on computing, Vol. 10, 276-286 (1998).

7. Andreatta, G., Brunetta, L.: Multiairport ground holding problem: a computational evaluation of exact algorithms. Operations Research, 46, 57-64 (1998).

8. Alonso-Ayuso, A., Escudero, L.F., Garín, A., Ortuño, M.T., Pérez, G.: An approach for strategic supply chain planning under uncertainty based on stochastic 0-1 programming. Journal of Global Optimization, 26, 97-124 (2003). 